\title{
Forkhead box A3 attenuated the progression of fibrosis in a rat model of biliary atresia
}

\author{
Rui Dong ${ }^{1,2}$, Yifan Yang ${ }^{1,2}$, Zhen Shen ${ }^{1}$, Chao Zheng ${ }^{1}$, Zhu Jin ${ }^{1}$, Yanlei Huang ${ }^{1}$, Zhien Zhang ${ }^{1}$, Shan Zheng ${ }^{*, 1}$ and Gong Chen ${ }^{\star, 1}$
}

Biliary atresia is a rare, devastating disease of infants where a fibroinflammatory process destroys the bile ducts, leading to fibrosis and biliary cirrhosis, and death if untreated. The cause and pathogenesis remain largely unknown. We tried to investigate factors involved in biliary atresia, especially forkhead box A3 (Foxa3), which might exert a role in the treatment of liver disease. We used RNA sequencing to sequence the whole transcriptomes of livers from six biliary atresia and six choledochal cysts patients. Then, we employed a rat disease model by bile duct ligation (BDL) and adenovirus transduction to address the function of Foxa3 in biliary atresia. We found that tight junction, adherence junction, cell cycle, apoptosis, chemokine singling, VEGF and MAPK signaling pathways were enriched in biliary atresia livers. We showed that Foxa3 expression was notably decreased in liver samples from biliary atresia patients. More importantly, we found that its lower expression predicted a poorer overall survival of biliary atresia patients. Rats that received BDL surgery and Foxa3 expression adenovirus resulted in a significant decrease in the deposition of collagen, and expression of profibrotic cytokines (transforming growth factor- $\beta$ and connective tissue growth factor) and fibrosis markers ( $\alpha$-smooth muscle actin, collagen I and collagen III), as compared with rats that received BDL surgery and control adenovirus. Our data suggested a protection role for Foxa3 during the progression of liver fibrosis in biliary atresia, and thereby supported increasing Foxa3 as a targeted treatment strategy.

Cell Death and Disease (2017) 8, e2719; doi:10.1038/cddis.2017.99; published online 30 March 2017

Biliary atresia is a common cause of neonatal cholestasis characterized by inflammation and bile duct obstruction. It causes cholestasis and progressive liver fibrosis and cirrhosis in infants. ${ }^{1-3}$ If left untreated, progressive liver cirrhosis leads to liver failure and death by age 2 years. Possible causes of biliary atresia have been proposed, including congenital malformation, congenital cytomegalovirus infection and reovirus 3 infection. ${ }^{4-6}$ Prognosis of biliary atresia has been significantly improved by Kasai operation, but the majority of patients still need a liver transplant to survive for a long time. ${ }^{7}$

Several studies have performed cDNA microarray to investigate the gene expression profiling of livers from biliary atresia patients. $^{8-10}$ However, microarrays do have several intrinsic limitations, such as narrow dynamic ranges, low specificity, low sensitivity and hybridization artifacts. ${ }^{11}$ Nextgeneration RNA sequencing (RNA-Seq) approach exhibits superior sensitivity and capability of detecting splice variants, thus remedying to the above limitations. ${ }^{12}$

In the current study, we used next-generation RNA-Seq to sequence the whole transcriptomes of livers from patients with biliary atresia or control (patients with choledochal cysts (CDCs)). The differential expressed genes were identified between these two groups and the results were then validated by qRT-PCR on several genes. Further, gene set enrichment analysis (GSEA) indicated that the upregulated genes were associated with tight junction, adherence junction, cell cycle, apoptosis, chemokine singling, VEGF and MAPK signaling pathways.

Forkhead box A3 (Foxa3, also known as hepatocyte nuclear factor $3 \gamma$ ) is a member of Foxa subfamily of forkhead box proteins, which can bind DNA through a conserved winged-helix- binding motif and act as transcriptional regulators. $^{13}$ Foxa subfamily proteins including Foxa1, Foxa2 and Foxa3 are of high importance in metabolism, organ development and differentiation. ${ }^{14,15}$ Although either perinatal or embryonic lethality was observed in Foxa1- and Foxa2-null mice, ${ }^{16,17}$ Foxa3-deficient mice are viable without any obvious abnormalities. ${ }^{18}$ Foxa family proteins are key transcriptional regulators during liver development. ${ }^{14} \mathrm{~A}$ recent study reported that overexpression of Foxa3 and hepatocyte nuclear factor $4 a$ (HNF4a) can convert rat bone marrow mesenchymal stem cells to functional hepatocyte-like cells, ${ }^{19}$ which indicates that Foxa3 might exert a role in the treatment of liver disease. Here, among the downregulated genes identified, Foxa3 was confirmed to be downexpressed in biliary atresia livers by western blot and immunohistochemical staining. Further investigation by bile duct ligation (BDL)-induced biliary atresia model indicated that ectopic expression of Foxa3 was significantly attenuated liver fibrosis. Based on our current findings, Foxa3 could be a promising target gene for biliary atresia therapy owing to its downregulation in biliary atresia livers.

${ }^{1}$ Department of Pediatric Surgery, Children's Hospital of Fudan University, and Key Laboratory of Neonatal Disease, Ministry of Health, Shanghai, China

*Corresponding author: S Zheng or G Chen, Department of Pediatric Surgery, Children's Hospital of Fudan University, and Key Laboratory of Neonatal Disease, Ministry of Health, 399 Wan Yuan Road, Shanghai 201102, China. Tel: +86 021 64931007; Fax: +86 021 64931901; E-mail: szheng@shmu.edu.cn or chengongzlp@ hotmail.com

${ }^{2}$ These authors contributed equally to this work.

Received 29.9.16; revised 06.1.17; accepted 14.2.17; Edited by A Oberst 


\section{Results}

Identification of differential expressed genes (DEGs) between biliary atresia and control liver tissues by RNA-seq analysis. Sixty patients (35 males and 25 females) with type III biliary atresia and 15 CDC patients were enrolled in this study. The CDCs patients with normal liver function were served as control group. Characteristics of the patients are listed in Supplementary Table S1. The serum levels of liver function-related enzymes (alkaline phosphatase, alanine transaminase, aspartate transaminase and gamma-glutamyl transpeptidase), total bile acid, total bilirubin and direct bilirubin were normal in these CDCs patients, but significantly increased in biliary atresia patients. These data revealed the impaired liver function and jaundice in biliary atresia patients. Moreover, representative HE staining and Masson's trichrome showed severe liver fibrosis and collagen accumulation in biliary atresia livers, as compared with control liver (Figure 1).

We then performed RNA-seq on six pairs of biliary atresia and control liver tissues using the Illumina platform. Genes that exhibited more than 1.5-fold differentially expressed with a $P$-value less than 0.05 were then defined as DEGs. Here, we identified a total of 1751 significantly DEGs with 772 upregulations (Supplementary Table S2) and 979 downregulations (Supplementary Table S3) in biliary atresia liver tissues, when compared with control tissues. The DEGs were functionally related with morphogenesis, fibrogenesis, tissue remodeling, metabolism, cell signal transduction, immunity and so forth. We then carried out GSEA to further determine which biological processes or pathways were involved in biliary atresia. Among 178 KEGG pathways, 31 and 44 pathways were enriched in biliary atresia liver tissues (Table 1) and control tissues (Table 2), respectively (nominal $P<0.05$ ). It is noteworthy that biliary atresia was positively correlated with multiple genes in tight junction, adherence junction, cell cycle, apoptosis, chemokine singling, VEGF and MAPK signaling pathways, while negatively correlated with multiple genes in bile acid biosynthesis. Validation analysis using
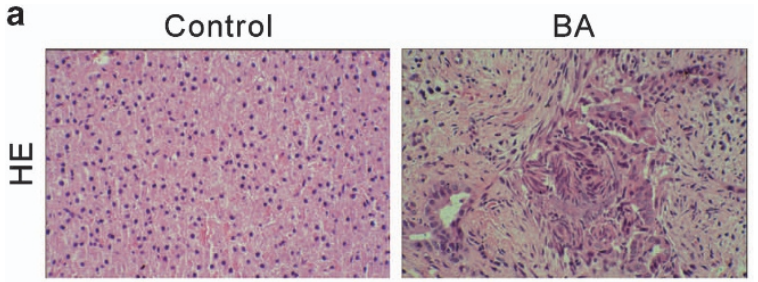

b
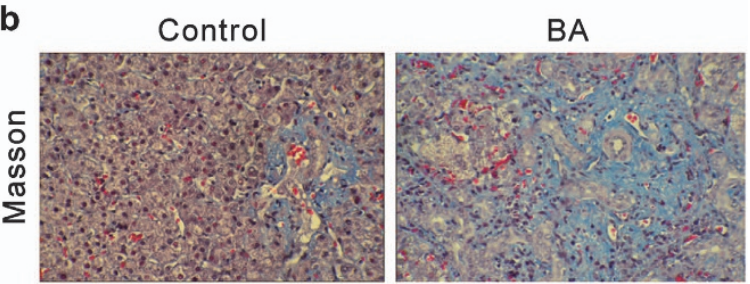

Figure 1 Histological analyses of livers from biliary atresia patients and control. (a) HE and (b) Masson's trichrome staining

Table 1 Statistically significant KEGG classifications of enrichment in biliary atresia patients

\begin{tabular}{|c|c|c|c|}
\hline KEGG subcategories & Size & $P$-value & ES \\
\hline FC_GAMMA_R_MEDIATED_PHAGOCYTOSIS & 96 & 0.0000 & 0.4904 \\
\hline CELLL_CYCLE & 124 & 0.0000 & 0.4553 \\
\hline PANCREATIC_CANCER & 70 & 0.0000 & 0.4754 \\
\hline UBIQUITIN_MEDIATED_PROTEOLYSIS & 132 & 0.0000 & 0.4414 \\
\hline PATHWAYS_IN_CANCER & 320 & 0.0010 & 0.3949 \\
\hline T_CELL_RECEPTOR_SIGNALING_PATHWAY & 107 & 0.0010 & 0.4608 \\
\hline SMALL_CELL_LUNG_CANCER & 84 & 0.0020 & 0.4556 \\
\hline ENDOMETRIĀL_CANCER & 52 & 0.0021 & 0.5053 \\
\hline COLORECTAL_CANCER & 62 & 0.0031 & 0.4647 \\
\hline HEDGEHOG_SIGNALING_PATHWAY & 53 & 0.0064 & 0.4819 \\
\hline AXON_GUIDĀNCE & 128 & 0.0080 & 0.3976 \\
\hline ADHERENS_JUNCTION & 73 & 0.0083 & 0.4452 \\
\hline NON_SMALL_CELL_LUNG_CANCER & 54 & 0.0096 & 0.4716 \\
\hline TIGHTT_JUNCTION & 128 & 0.0101 & 0.4003 \\
\hline ACUTE__MYELOID_LEUKEMIA & 57 & 0.0105 & 0.4580 \\
\hline SPLICEOSOME & 125 & 0.0121 & 0.3977 \\
\hline CHRONIC_MYELOID_LEUKEMIA & 73 & 0.0145 & 0.4225 \\
\hline PHOSPHATIDYLINOSITOL_SIGNALING_SYSTEM & 75 & 0.0185 & 0.4160 \\
\hline NEUROTROPHIN_SIGNALING_PATHWAY & 124 & 0.0212 & 0.3798 \\
\hline CHEMOKINE_SIGNALING_PATHWAY & 180 & 0.0261 & 0.3565 \\
\hline HOMOLOGOŪS_RECOMBINNATION & 28 & 0.0284 & 0.5023 \\
\hline BASAL_CELL_CARCINOMA & 53 & 0.0335 & 0.4334 \\
\hline ERBB_SIGNALING_PATHWAY & 87 & 0.0372 & 0.3939 \\
\hline APOPTTOSIS & 84 & 0.0379 & 0.3974 \\
\hline THYROID_CANCER & 29 & 0.0394 & 0.4947 \\
\hline NATURAL_KILLER_CELL_MEDIATED_CYTOTOXICITY & 118 & 0.0394 & 0.3762 \\
\hline VEGF_SIGNALING_PATHWAY & 72 & 0.0404 & 0.4117 \\
\hline MAPK_SIGNALING_PATHWAY & 256 & 0.0410 & 0.3409 \\
\hline BASAL_TRANSCRIPTION_FACTORS & 33 & 0.0416 & 0.4796 \\
\hline LEISHMANIA_INFECTION & 70 & 0.0487 & 0.4022 \\
\hline LEUKOCYTE_TRANSENDOTHELIAL_MIGRATION & 110 & 0.0491 & 0.3799 \\
\hline
\end{tabular}


Table 2 Statistically significant KEGG classifications of enrichment in normal control

\begin{tabular}{|c|c|c|c|}
\hline KEGG subcategories & Size & $P$-value & ES \\
\hline RETINOL_METABOLISM & 61 & 0.0000 & -0.6814 \\
\hline METABOLISM_OF_XENOBIOTICS_BY_CYTOCHROME_P450 & 65 & 0.0000 & -0.6900 \\
\hline FATTY_ACID_METABOLISM & 42 & 0.0000 & -0.7198 \\
\hline DRUG_METABBOLISM_CYTOCHROME_P450 & 67 & 0.0000 & -0.6611 \\
\hline VALINE_LEUCINE_AND_ISOLEUCINE_DEGRADATION & 44 & 0.0000 & -0.6638 \\
\hline RIBOSOME & 85 & 0.0000 & -0.5872 \\
\hline COMPLEMENT_AND_COAGULATION_CASCADES & 67 & 0.0000 & -0.5820 \\
\hline PRIMARY_BILE_ACID_BIOSYNTHESIS & 16 & 0.0000 & -0.8517 \\
\hline STEROID_HORMONE_BIOSYNTHESIS & 52 & 0.0000 & -0.6167 \\
\hline OXIDATIVE_PHOSPHORYLATION & 112 & 0.0000 & -0.5179 \\
\hline PROPANOĀTE_METABOLISM & 32 & 0.0000 & -0.6790 \\
\hline TRYPTOPHAN_METABOLISM & 39 & 0.0000 & -0.6453 \\
\hline PARKINSONS DISEASE & 110 & 0.0000 & -0.5182 \\
\hline PEROXISOMĒ & 78 & 0.0000 & -0.5082 \\
\hline LINOLEIC_ACID_METABOLISM & 26 & 0.0000 & -0.6252 \\
\hline GLYCINE SERINE AND THREONINE METABOLISM & 31 & 0.0000 & -0.6368 \\
\hline TYROSINE_METABOLISM & 39 & 0.0000 & -0.5651 \\
\hline GLYCOLYSIS_GLUCONEOGENESIS & 59 & 0.0000 & -0.4957 \\
\hline GLYOXYLATE AND DICARBOXYLATE METABOLISM & 15 & 0.0000 & -0.7207 \\
\hline PENTOSE_AND_GLUCURONATE_INTERCONVERSIONS & 26 & 0.0000 & -0.6133 \\
\hline PROTEASŌME & 44 & 0.0000 & -0.5106 \\
\hline DRUG_METABOLISM_OTHER_ENZYMES & 49 & 0.0000 & -0.4956 \\
\hline PORPHYRIN_AND_CHLOROPHYLL_METABOLISM & 38 & 0.0000 & -0.5217 \\
\hline ASCORBATE_AND_ALDARATE_METABOLISM & 23 & 0.0000 & -0.5724 \\
\hline BETA_ALANIN̄E_MËTABOLISM ${ }^{-}$ & 21 & 0.0000 & -0.5847 \\
\hline PPAR_SIGNALING_PATHWAY & 65 & 0.0000 & -0.4127 \\
\hline ARACHIDONIC_ACID_METABOLISM & 55 & 0.0000 & -0.4299 \\
\hline PYRUVATE_METABOL̄ISM & 39 & 0.0000 & -0.4370 \\
\hline TERPENOID BACKBONE BIOSYNTHESIS & 15 & 0.0000 & -0.5692 \\
\hline RENIN_ANGIOOTENSIN_SȲSTEM & 16 & 0.0000 & -0.5532 \\
\hline ONE_CARBON_POOL_BY_FOLATE & 17 & 0.0000 & -0.5381 \\
\hline BUTANNOATE_METABOLISM & 32 & 0.0000 & -0.4446 \\
\hline HUNTINGTONS_DISEASE & 167 & 0.0000 & -0.4495 \\
\hline CITRATE_CYCLEE_TCA_CYCLE & 29 & 0.0000 & -0.3206 \\
\hline ARGININE_AND_PROLINNE_METABOLISM & 54 & 0.0000 & -0.4296 \\
\hline ALZHEIMERS DISEASE & 154 & 0.0000 & -0.3626 \\
\hline GLUTATHIONĒ_METABOLISM & 48 & 0.0000 & -0.3144 \\
\hline PRION_DISEASES & 34 & 0.0000 & -0.3474 \\
\hline NEUROACTIVE_LIGAND_RECEPTOR_INTERACTION & 222 & 0.0000 & -0.3628 \\
\hline PENTOSE_PHOSPHATE_PATHWAY & 26 & 0.0078 & -0.3808 \\
\hline HISTIDINE_METABOLISM & 28 & 0.0263 & -0.3546 \\
\hline NICOTINATE_AND_NICOTINAMIDE_METABOLISM & 24 & 0.0348 & -0.3149 \\
\hline STARCH_AND_SUCEROSE_METABOLISM & 46 & 0.0357 & -0.3833 \\
\hline PYRIMIDINNE_M̄ETABOLISM̄ & 98 & 0.0417 & -0.2991 \\
\hline
\end{tabular}

qRT-PCR for four DEGs demonstrated the consistent overexpression for connective tissue growth factor (CTGF) and death-associated protein kinase 1 (DAPK1), and the consistent under-expression for Foxa3 and epidermal growth factor like domain 7 (EGFL7) in biliary atresia livers (Figure 2a).

Furthermore, comparing with control livers, a significant increase in protein level of CTGF and a notable decrease in protein level of Foxa3 were observed in biliary atresia livers as measured by western blot (Figure $2 b$ ) and immunohistochemistry staining (Figure 2c).

Downregulation of Foxa3 was associated with the overall survival of biliary atresia. To further explore the functions of Foxa3 on biliary atresia in vivo, we established a rat biliary atresia model by BDL. Firstly, we performed RNA-seq on four pairs of BDL and sham-operated liver tissues. A total of 2618 significantly DEGs with 1667 upregulations (Supplementary Table S4) and 951 downregulations (Supplementary Table S5) were identified in BDL liver tissues, when compared with sham-operated liver tissues. We found that 44 DEGs were upregulated, while 62 DEGs were downregulated in both liver samples from biliary atresia patients and BDL rats (Supplementary Figure S1). Four transcription factors, HMGB2, STOH8, CEBPB and Foxa3, were included in the 106 DEGs (Supplementary Table S6). Foxa3 is a member of Foxa family proteins, ${ }^{20}$ which are key transcriptional regulators during liver development. ${ }^{14}$ Overexpression of Foxa3 and HNF4a can convert rat bone marrow mesenchymal stem cells to functional hepatocyte-like cells. ${ }^{19}$ Thus, we chose Foxa3 for further investigation.

To evaluate the possible prognostic value of Foxa3 on biliary atresia, we detected Foxa3 mRNA expression on all 60 biliary atresia liver samples by qRT-PCR, and divided the patients into Foxa3-high expression group and Foxa3-low expression group by using the median value of Foxa3 mRNA level as a cut-off. We then carried out Kaplan-Meier survival analysis of biliary atresia cases to investigate the clinical outcome of each group. Low expression of Foxa3 was 
a
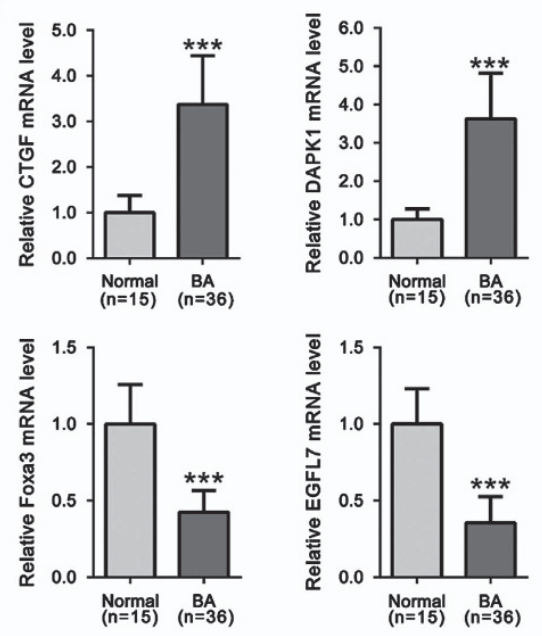

b
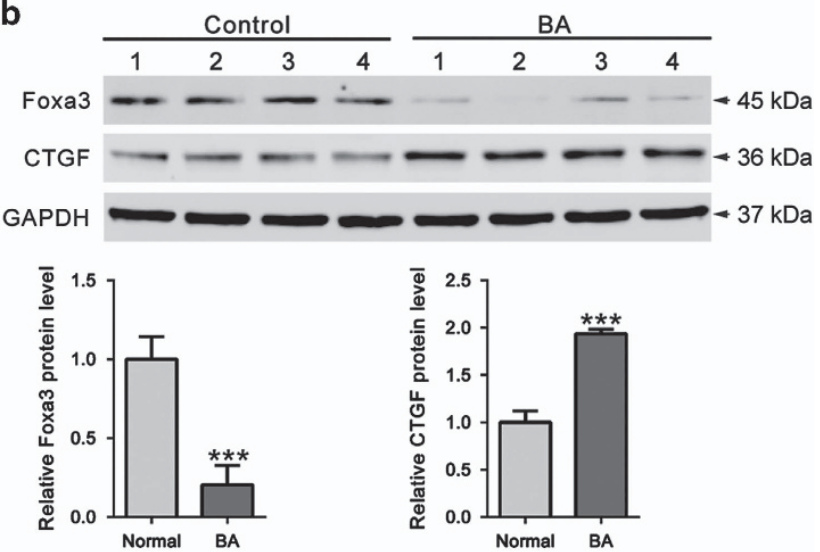

C
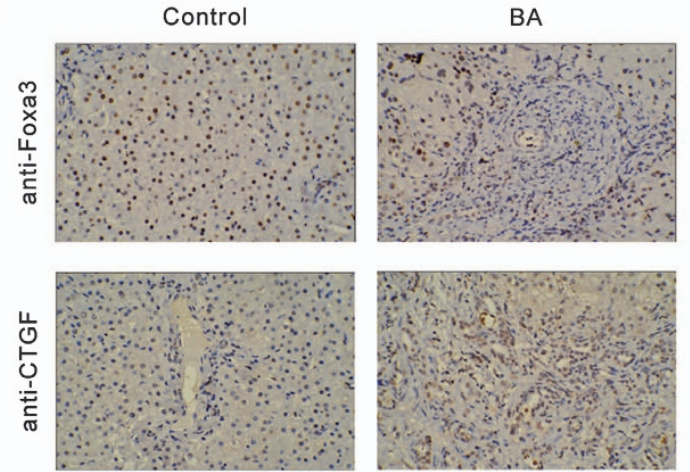

Figure 2 Validation of RNA-seq data. (a) mRNA levels of CTGF, DAPK1, Foxa3 and EGFL7 were evaluated by qRT-PCR. Data represent mean values \pm S.D. from three independent experiments. Protein levels of Foxa3 and CTGF in livers of biliary atresia patients and control were assessed by western blotting (b) and immunohistochemistry staining (c) ${ }^{* * *} P<0.001$ versus control)

associated with poor overall survival of biliary atresia patients (log-rank $=8.379, \quad P=0.0038$ ) (Figure 3). By regression analysis, we found that Foxa3 expression had a negative correlation with the degree of fibrosis $(P<0.001$, coefficient of determination $R=-0.7398$ ), while no obvious correlation was found between age and Foxa3 expression $(P>0.05)$.

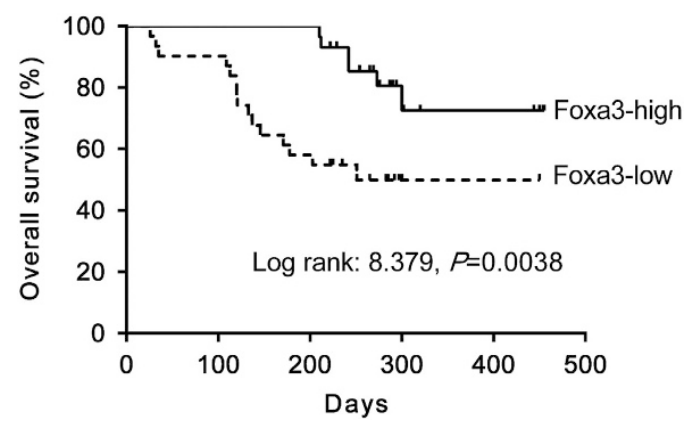

Figure 3 Downregulation of Foxa3 was associated with the overall survival of biliary atresia. Overall survival analysis on 60 biliary atresia patients was performed using the Kaplan-Meier method and log-rank tests

Ad5-Foxa3 transduction remarkably attenuated fibrosis in a rat BDL model. To further emphasize the specific effects of Foxa3, Foxa3-expressing adenoviruses (Ad5Foxa3) were generated and delivered to the rat BDL model by tail-vein injection 2 weeks after surgery. Four weeks after surgery, liver tissues were collected and western blot was carried out to evaluate Foxa3 expression. Supplementary Figure S2 showed that Foxa3 expression was significantly suppressed by BDL surgery, and rescued by Ad5-Foxa3 injection (Figure $4 \mathrm{a}$ ). In rats receiving BDL surgery and control adenovirus (Ad5) injection, liver fibrosis and extensive collagen deposition was evident, as shown by HE (Figure 4b) and Masson's trichrome staining (Figure 4c). Injection of Ad5-Foxa3 largely inhibited hepatic collagen accumulation after BDL. These data indicated that Foxa3 can alleviate the fibrosis induced by BDL. The mRNA and protein levels of profibrotic cytokines (TGF- $\beta 1$ and CTGF) and fibrosis markers ( $\alpha$-SMA, Collagen I and Collagen III) were then detected (Figure 5). Comparing with sham-operated rats, all detected cytokines and fibrosis markers were significantly increased in livers of BDL rats, which were notably reduced by Ad5-Foxa3 injection. These data suggested the protection effects of Foxa3 on liver fibrosis.

Expression of Foxa3 and CTGF in rhesus rotavirus (RRV)-induced experimental biliary atresia. As a BDL model in rats has little similarity to the biliary atresia, we also established a mouse biliary atresia model by injection with RRV. As shown in Figure 6a, RRV induced severe liver fibrosis and collagen accumulation, as compared with control livers. The changes of Foxa3 and CTGF protein expression were consistent with results observed in human samples.

\section{Discussion}

In this study, we conducted RNA sequencing for six biliary atresia and six CDCs liver tissues. These CDCs patients showed normal liver function as assessed by serum indices and histological analysis, and were served as control in the present study. We analyzed the expression difference at gene levels between BA and control liver tissues and identified 1751 DEGs (Supplementary Tables S2 and S3), many of which were identified as DEGs in previous studies, such as CFTR (cystic fibrosis transmembrane conductance regulator), 
a
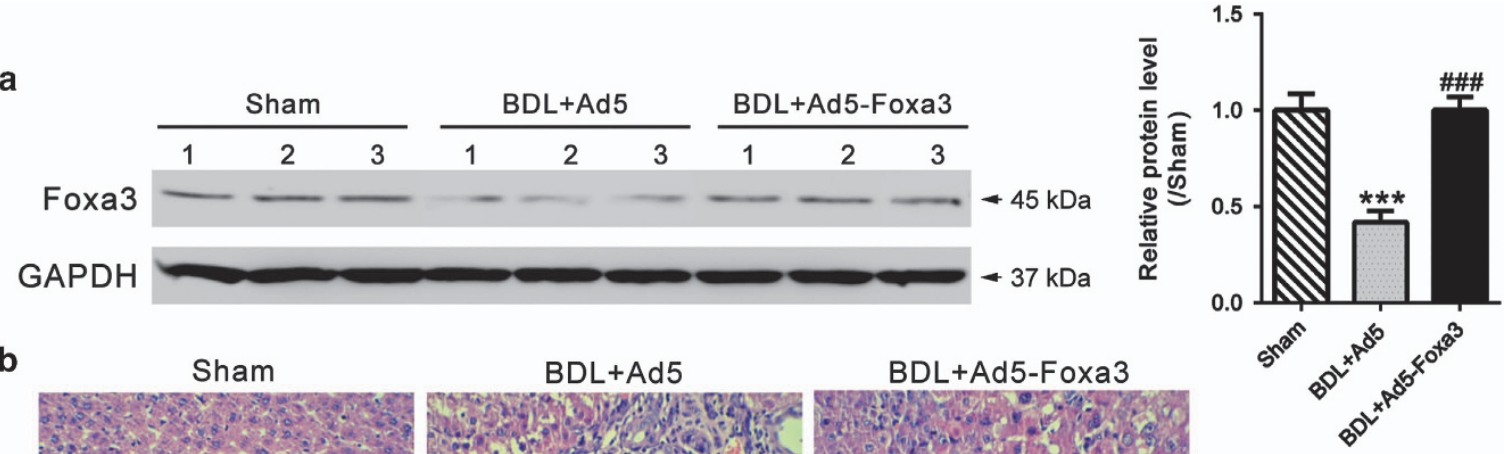

b

Sham

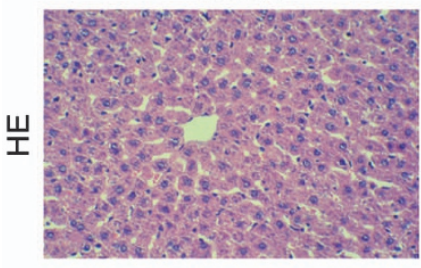

C

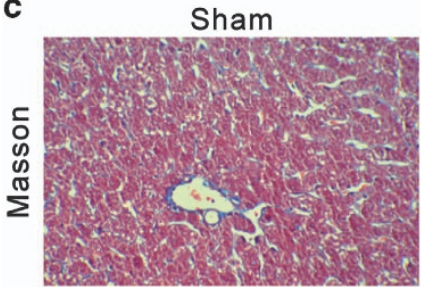

$B D L+A d 5$

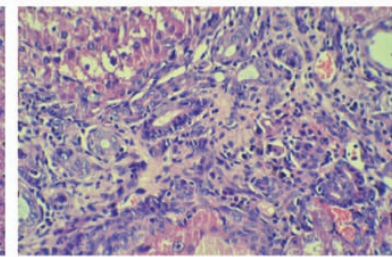

$B D L+A d 5$

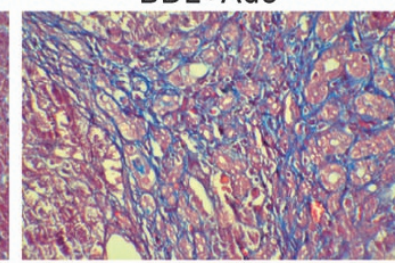

BDL+Ad5-Foxa3

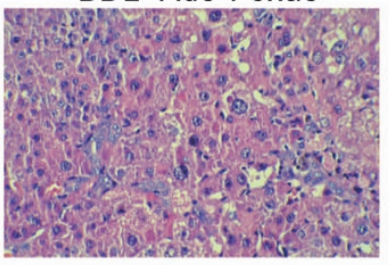

BDL+Ad5-Foxa3

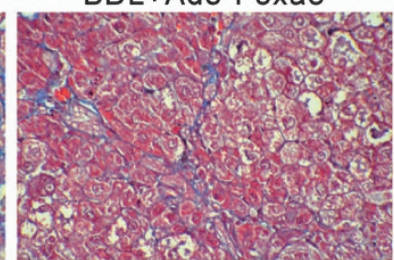

Figure 4 Effect of Ad5-Foxa3 treatment on BDL-induced liver fibrosis. Rats received sham operation or bile duct ligation (BDL) as described in Material and Methods. Two weeks later, rats undergoing BDL surgery were injected with Foxa3-expressing adenovirus (Ad5-Foxa3) or control adenovirus (Ad5). After 2 more weeks, liver tissues were collected. (a) Foxa3 expression was evaluated by western blot analysis. Histological analyses were performed by HE (b) and Masson's trichrome staining (c). BDL surgery caused severe liver fibrosis, while Ad5-Foxa3 injection significantly attenuated BDL-induced liver fibrosis ( ${ }^{\star \star \star} P<0.001$ versus sham-operated group; ${ }^{\# \# \# ~} P<0.001$ versus BDL + Ad5 group)

MMP-7 (matrix metalloproteinase-7), CTGF, LAMC2 (laminin, gamma 2) and VTCN1 (V-set domain containing T-cell activation inhibitor 1). ${ }^{8,21-23}$ We also identified additional novel DEGs, suggesting that the RNA-seq based approach is extremely powerful to study expression profiling.

Biliary atresia is a severe chronic cholestasis disorder of infants. Distinct plasma bile acid profiles were reported in biliary atresia patients. ${ }^{24}$ Recently, increasing evidence has liked the Foxa family of transcription factors, which take part in metabolism, organ development and differentiation, ${ }^{14}$ to bile acid metabolism. Foxa1 and Foxa2 are required for the development of normal bile duct through preventing excess cholangiocyte proliferation. ${ }^{25}$ Bochkis et al. reported that hepatocyte-specific knockout of Foxa2 decreased transcription of genes encoding bile acid transporters, resulting in intrahepatic cholestasis. They also found that Foxa2 was markedly reduced in pediatric subjects with primary sclerosing cholangitis and in those with biliary atresia. ${ }^{26}$ In this study, Foxa3 was identified as a downregulated gene in biliary atresia livers. Our data showed that Foxa3 was notably decreased in liver samples from biliary atresia patients as evaluated by qRT-PCR, western blotting and immunohistochemistry staining (Figure 2). These findings were further confirmed in BDL (Supplementary Figure S2) and RRVinduced experimental biliary atresia (Figure 6). More importantly, we found that lower expression of Foxa3 predicted a poorer overall survival of biliary atresia patients (Figure 3 ). We then used the BDL model to explore whether Foxa3 is relevant to the mechanisms of this disease. We found that Foxa3 expressing adenovirus transduction significantly weakened liver fibrosis induced by BDL (Figures 4 and 5). Our data suggested that Foxa3 was a potential prognosis factor for biliary atresia and it may exert antifibrotic effects during the pathogenesis of this disease. However, further investigation is required to figure out how Foxa3 influences profibrotic cytokines and fibrosis markers or whether Foxa3 affects transcription of genes encoding bile acid transporters.

In summary, we reported the expression profile of biliary atresia, and indicated the clinical value of Foxa3 in patients with biliary atresia although there is still long way to go before it can be applied to the clinic.

\section{Materials and Methods}

Patient samples. This study was approved by the Human Ethics Boards at Children's Hospital of Fudan University. Written informed consent was obtained from the legal guardians of all subjects before starting study procedures. Sixty patients with type III biliary atresia (including 5 patients with advanced cirrosisis) and 15 patients with CDCs who were treated at Children's Hospital of Fudan University from January 2014 to July 2014 were enrolled in this study. Patients with syndromic biliary atresia were excluded. The clinical characteristics of the patients are presented in Supplementary Table S1. All biliary atresia patients underwent successful Kasai portoenterostomy and follow-up lasted for one year. The age at diagnosis was $2.1 \pm 0.6$ months. None of the patients received liver transplant within one year after surgery. All the samples collected at the time of Kasai portoenterostomy. For RNA and protein extraction, tissue samples were immediately 
a
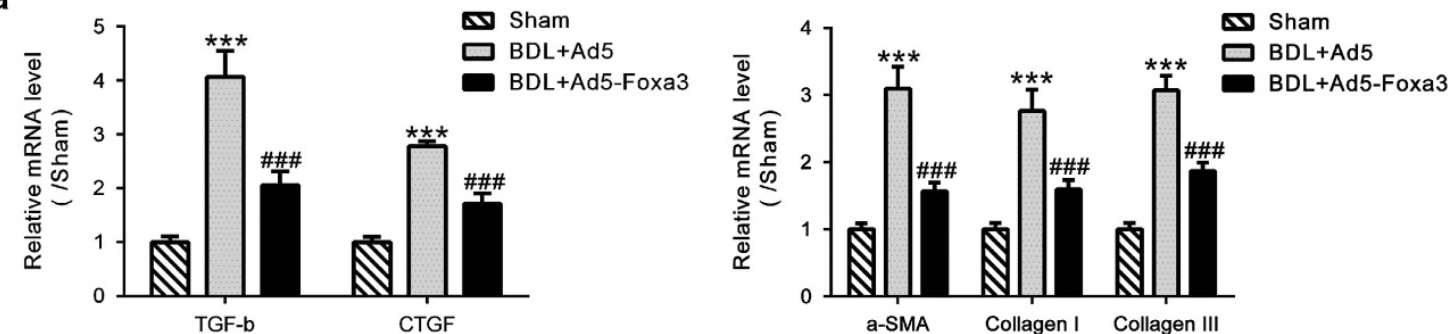

b
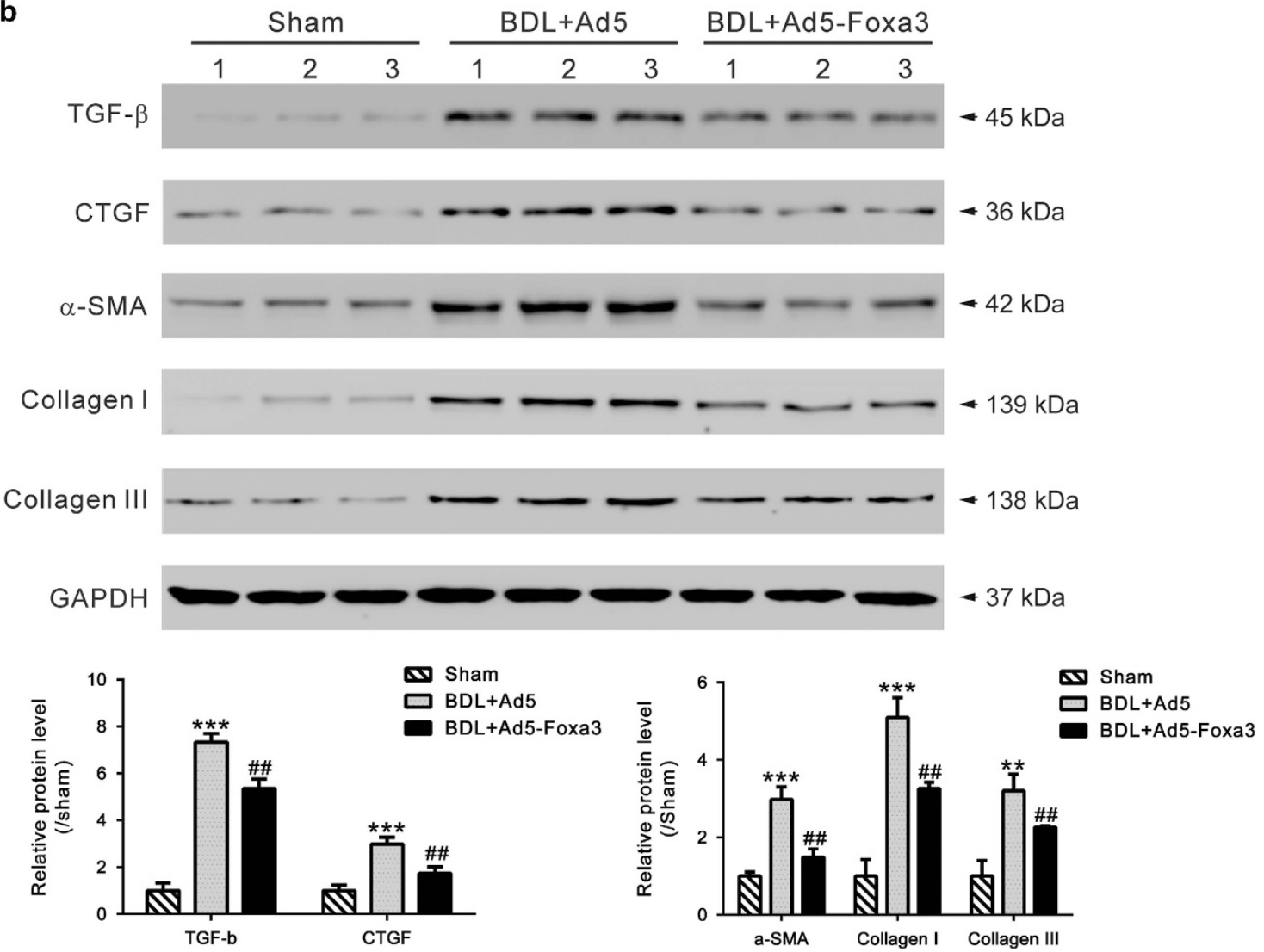

Figure 5 Effect of Ad5-Foxa3 treatment on expression of profibrotic cytokines and fibrosis markers. (a) Hepatic mRNA and (b) protein levels of TGF- $\beta 1$, CTGF, $\alpha$-SMA, Collagen I and Collagen III were assessed by qRT-PCR and western blotting, respectively $\left({ }^{* *} P<0.01\right.$ versus sham-operated group, ${ }^{* * *} P<0.001$ versus sham-operated group; ${ }_{\# \#} P<0.01$ versus $B D L+A d 5$ group,, ${ }^{\# \# \# ~} P<0.001$ versus $B D L+A d 5$ group). Data represent mean values \pm S.D. from three independent experiments

snap-frozen and stored at $-80^{\circ} \mathrm{C}$. Liver biopsies were scored for fibrosis stage according to the Metavir score system, which classifies fibrosis according to a 5-point scale: F0, no fibrosis; F1, portal fibrosis without septa; F2, portal fibrosis with few septa; F3, numerous septa without cirrhosis; F4, cirrhosis. Regression analysis was carried out to determine the relationship between the degree of fibrosis and Foxa3 by using Medcalc software (MedCalc, Ostend, Belgium).

RNA extraction, processing and sequencing. Total RNA was extracted from liver tissues using the Trizol reagent (Invitrogen, Carlsbad, CA, USA) according to the manufacturer's protocol. Extracted RNA was quantified by using an ND-1000 Spectrophotometer (NanoDrop Technologies, Wilmington, DE, USA). RNA integrity was assessed by denaturing formaldehyde gel electrophoresis. RNA-Seq libraries were prepared by using Illumina's TruSeq Sample Preparation Kit. Briefly, polyA-containing messenger RNA was captured from $10 \mu \mathrm{g}$ of total RNA, fragmented into small fragments, and reverse-transcribed into cDNA. The cDNA was fragmented and ligated to adapters. The cDNA libraries was then created by using 15 cycles of PCR. Each sample was cleaned up on an RNeasy Mini
Column (Qiagen, Limburg, Netherlands), treated with DNase and analyzed for quality on an Agilent 2100 Bioanalyzer (Agilent Technologies, Santa Clara, CA, USA). Samples were on an Illumina HiSeq 2000 for $2 \times 100$-bp paired-end sequencing. Reads were mapped to the human genome (hg19) using TopHat v2.0.11 (http://tophat.cbcb.umd.edu) ${ }^{27}$ with the following default options with a TopHat transcript index built from Ensembl_GRCh37. Transcript expression was estimated with an improved version of Cuffdiff2 (http://cufflinks.cbcb.umd.edu) ${ }^{28}$ Cuffdiff was run with the default options against the UCSC iGenomes GTF file from Illumina (available at http://cufflinks.cbcb.umd.edu/igenomes.html). The workflow used to analyze the data is described in detail in Trapnell et al. ${ }^{29}$ To identify a gene or transcript as differential expression, Cuffdiff2 tests the observed log-fold-change in its expression against the null hypothesis of no change (i.e., the true log-foldchange is zero). Clustering of gene expression profiles was achieved with the csDendro function from CummeRbund (http://compbio.mit.edu/cummeRbund/).

All of our original sequence data have been deposited in NCBI's Sequence Read Archive database (http://www.ncbi.nlm.nih.gov/sra, AC: SRA297629 and SRP063995). 
a
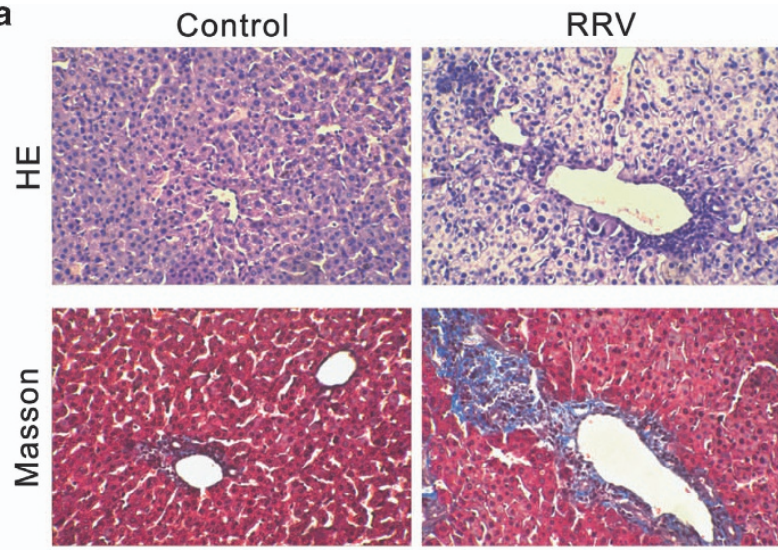

b
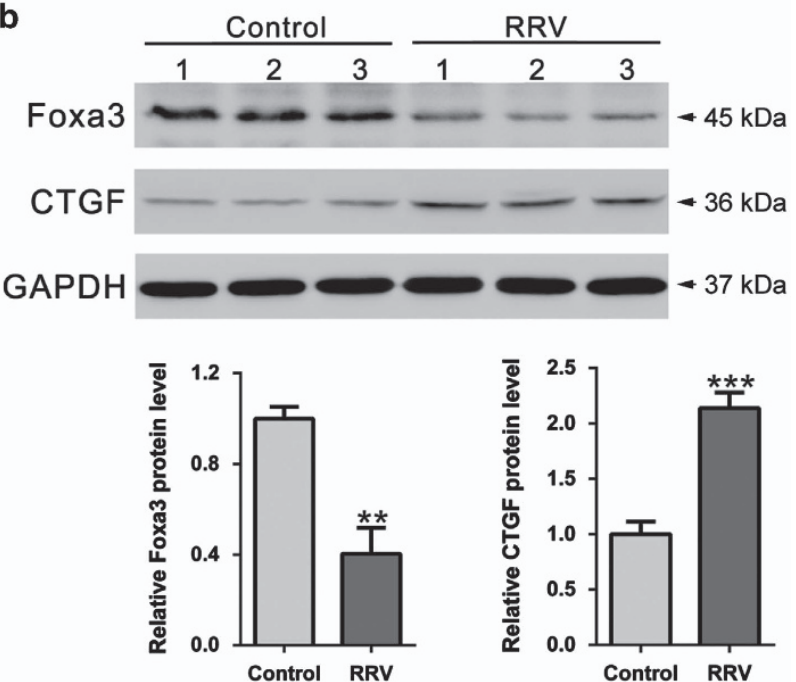

Figure 6 Expression of Foxa3 and CTGF in rhesus rotavirus (RRV)-induced experimental biliary atresia. (a) Histological analyses by HE or Masson's trichrome staining. (b) Foxa3 and CTGF expression was evaluated by western blot analysis $\left({ }^{\star \star} P<0.01,{ }^{* \star *} P<0.001\right.$ versus Control group)

Pathway analysis and bioinformatics. To identify the pathways that were significantly enriched in samples from biliary atresia patients or controls, GSEA was performed as describe previously ${ }^{30}$ using a total of 178 gene sets from Kyoto Encyclopedia of Genes and Genomes (KEGG).The gene sets showing FDR, 0.25, a well-established cutoff for the identification of biologically relevant genes, were considered enriched between the classes under comparison.

Histology and immunohistochemistry. Liver specimens were fixed in $10 \%$ neutral-buffered formalin and embedded in paraffin. The embedded tissues were cut into $5 \mu \mathrm{m}$-thick serial sections. The sections were deparaffinized in xylene, hydrated through graded ethanol and stained with hematoxylin \& eosin (HE) or Masson's trichrome. For immunohistochemistry staining, deparaffinized and hydrated sections were rinsed in PBS and treated with $0.3 \%$ hydrogen peroxide for blocking endogenous peroxidase activity. After probed with primary antibodies (antibodies against CTGF and Foxa3 were from Abcam (Cambridge, UK) and Santa Cruz (Santa Cruz, CA, USA), respectively) at $4{ }^{\circ} \mathrm{C}$ overnight, the sections were incubated with appropriate horseradish peroxidase-conjugated secondary antibodies (Longisland Biotech., Shanghai, China) at room temperature for $1 \mathrm{~h}$, and visualized with $\mathrm{DAB}$ substrate (Longisland Biotech.) followed by counterstain with hematoxylin. The optimal antibody concentration was determined for each assay with a titration experiment. Negative controls were performed with primary antibody absent and mouse or rabbit isotype control antibody.
Quantitative RT-PCR analysis. Total RNA was isolated from snap-frozen liver tissues using TRIzol Reagent (Invitrogen) followed by DNase treatment (Promega, Madison, WI, USA). Complementary DNA was prepared by reverse transcription with M-MLV reverse transcriptase (Fermentas, Hanover, MD, USA) according to the manufacturers' instructions. Quantitative RT-PCR was performed on an ABI 7300 Sequence Detection System (Applied Biosystems, Foster City, CA, USA) with SYBR Green PCR mix (Thermo Fisher Scientific, Rockford, IL, USA). Primers used are summarized in Supplementary Table S7. Gene expression values were calculated using the $\Delta \Delta \mathrm{Ct}$ method $^{31}$ and GAPDH was served as an endogenous control.

Western blotting. Snap-frozen liver samples were homogenized in radioimmunoprecipitation assay buffer. Protein concentration was determined by BCA protein assay (Thermo Fisher Scientific). Equal amounts of protein were subjected to SDS-PAGE gels following with electrophoretic transfer to nitrocellulose membranes. The membranes were blocked with $5 \%$ nonfat milk, and incubated with the primary antibody for Foxa3 (sc-25357; Santa Cruz), transforming growth factor- $\beta$ (TGF- $\beta$, Ab64715; Abcam), CTGF (Ab6992; Abcam), Collagen I (Ab34710; Abcam), Collagen III (Ab7778; Abcam), $\alpha$-Smooth muscle actin ( $\alpha$-SMA, \#14968; Cell Signaling Technology, Danvers, MA, USA) or GAPDH (\#5174, Cell Signaling Technology). After incubated with corresponding horseradish peroxidase-coupled secondary antibody (Beyotime, Shanghai, China), the membrane was developed with enhanced chemiluminescence system (Bio-Rad, Richmond, CA, USA). Densitometric analysis was performed by using ImageJ software (National Institutes of Health, Bethesda, MD, USA) using GAPDH as an endogenous control.

Construction of the recombinant adenovirus Ad5-Foxa3. Foxa3 expression plasmid (PDC315-Foxa3) and a LacZ-containing control plasmid (PDC315-LacZ) were constructed and confirmed by sequencing. PDC315-Foxa3 or PDC315-LacZ was then transfected into HEK293 cells with pBGHE3 (Microbix Biosystems, Mississauga, Ontario, Canada) by using Lipofectamine 2000 (Invitrogen) according to the protocol provided by the manufacturer. Recombinant adenovirus Ad5-Foxa3 or Ad5-LacZ was grown in HEK293 cells and purified on a cesium chloride gradient. After viral titers were determined by plaque assay, virus was aliquoted and stored at $-80^{\circ} \mathrm{C}$.

Rat disease model induced by BDL.The experiments were carried out according to the guidelines of the Ethics Committee of Fudan University. Six-week-old male Sprague-Dawley rats, weighing between 180 and $200 \mathrm{~g}$, were purchased from Shanghai Lab Animal Research Center (Shanghai, China) and kept on the same diet. Eighteen rats were randomly divided into three groups: group I, sham operated (control); group II, BDL+Ad5-LacZ; group III, BDL+Ad5-Foxa3. All surgical procedure was performed under strict sterile conditions as previously described. ${ }^{32}$ All rats were anesthetized by intraperitoneal injection with pentobarbital sodium $(50 \mathrm{mg} / \mathrm{kg})$. Laparotomy was performed on all rats via an approximately $1 \mathrm{~cm}$ upper-midline incision to identify the bile duct. In group II and group III, the common bile duct was isolated, doubly ligated and transected between the ligatures. In group I, no ligation or resection was performed. Two weeks after BDL, recombinant adenovirus Ad5-LacZ and Ad5-Foxa3 $\left(5 \times 10^{9} \mathrm{PFU}\right.$ per rat, $\left.0.5 \mathrm{ml}\right)$ were tail-vein injected into rats of group II and group III, respectively. Four weeks after surgery, rats were killed at 0900-0930 hours and killed, and liver tissues were then collected. For histological analysis, liver samples were fixed in $10 \%$ buffered formalin, embedded in paraffin and cut into $5-\mu \mathrm{m}$ slices; for RNA or protein extraction, liver samples were snap-frozen and stored at $-80^{\circ} \mathrm{C}$.

Mouse biliary atresia model induced by RRV. Healthy Balb/c pregnant maternal mice were purchased from SLAC Inc. (Shanghai, China) and isolated in laminar-flow cages. Newborn Balb/c mice were randomized into two groups in proportion of 2:1 (experimental group:control group). Pups in the experimental group were inoculated intraperitoneally with $25 \mu \mathrm{l}$ minimum essential medium, containing $10^{6} \mathrm{PFU}$ of RRV. Control group received $25 \mu \mathrm{l} 2 \%$ FCS-MEM. Pups that died due to infection, did not feed or that were cannibalized by their mothers were excluded from further analysis. All mice were weighed every 2 days and observed for signs of cholestasis (icterus of the non-fur-covered skin, color and quality of stools, and the appearance of bilirubin in the urine) until 14 days when they were killed. Liver samples were collected for histological analysis and western blotting analysis.

Statistical analysis. Statistical analyses were performed in GraphPad Prism v6.0 software (GraphPad Software Inc., La Jolla, CA, USA). Data were expressed as mean \pm S.D. For survival analysis, overall survival was defined as the time 
interval between the date of operation to the date of death or the last follow-up. The prognostic significance analysis was performed using the Kaplan-Meier method and log-rank tests. Statistical significance between two groups was determined by Student's $t$-test. Statistically significant differences were defined as having a $P$-value less than 0.05 .

\section{Conflict of Interest}

The authors declare no conflict of interest.

Acknowledgements. This study received financial support from National Key Clinical Specialty Construction Programs of China (2014-2016), Shanghai 'Non keyin-key discipline' Clinical medical centers (2014-2016), Shanghai Hospital Development Center (SHDC12014106), National Natural Science Foundation of China (no. 81370472, no. 81300517, no. 81401243 and no. 81500394), Shanghai Rising-Star Program (A type) (no. 15QA1400800) and The Science Foundation of Shanghai (no. 16411952200, no. 16140902300, no. 14ZR1404000 and no. 14411969860).

1. Hartley JL, Davenport M, Kelly DA. Biliary atresia. Lancet 2009; 374: 1704-1713.

2. Bassett MD, Murray KF. Biliary atresia: recent progress. J Clin Gastroenterol 2008; 42: 720-729.

3. Lee JY, Lim LT, Quak SH, Prabhakaran K, Aw M. Cholangitis in children with biliary atresia: health-care resource utilisation. J Paediatr Child Health 2014; 50: 196-201.

4. Mahjoub F, Shahsiah R, Ardalan FA, Iravanloo G, Sani MN, Zarei A et al. Detection of Epstein Barr virus by chromogenic in situ hybridization in cases of extra-hepatic biliary atresia. Diagn Pathol 2008; 3: 19.

5. Amer OT, Abd El-Rahma HA, Sherief LM, Hussein HF, Zeid AF, Abd El-Aziz AM. Role of some viral infections in neonatal cholestasis. Egypt J Immunol 2004; 11: 149-155.

6. Nakamura K, Tanoue A. Etiology of biliary atresia as a developmental anomaly: recent advances. J Hepatobiliary Pancreat Sci 2013; 20: 459-464.

7. Zagory JA, Nguyen MV, Wang KS. Recent advances in the pathogenesis and management of biliary atresia. Curr Opin Pediatr 2015; 27: 389-394.

8. Bezerra JA, Tiao G, Ryckman FC, Alonso M, Sabla GE, Shneider B et al. Genetic induction of proinflammatory immunity in children with biliary atresia. Lancet 2002; 360: 1653-1659.

9. Leonhardt J, Stanulla M, von Wasielewski R, Skokowa J, Kubler J, Ure BM et al. Gene expression profile of the infective murine model for biliary atresia. Pediatr Surg Int 2006; 22 84-89.

10. Chen L, Goryachev A, Sun J, Kim P, Zhang H, Phillips MJ et al. Altered expression of genes involved in hepatic morphogenesis and fibrogenesis are identified by cDNA microarray analysis in biliary atresia. Hepatology 2003; 38: 567-576.

11. Hurd PJ, Nelson CJ. Advantages of next-generation sequencing versus the microarray in epigenetic research. Brief Funct Genomic Proteomic 2009; 8: 174-183.

12. Costa V, Aprile M, Esposito R, Ciccodicola A. RNA-Seq and human complex diseases: recent accomplishments and future perspectives. Eur J Hum Genet 2013; 21: 134-142.

13. Hannenhalli S, Kaestner KH. The evolution of Fox genes and their role in development and disease. Nat Rev Genet 2009; 10: 233-240.

14. Friedman JR, Kaestner KH. The Foxa family of transcription factors in development and metabolism. Cell Mol Life Sci 2006; 63: 2317-2328.

15. Kaestner KH. The FoxA factors in organogenesis and differentiation. Curr Opin Genet Dev 2010; 20: 527-532.

16. Kaestner KH, Katz J, Liu Y, Drucker DJ, Schutz G. Inactivation of the winged helix transcription factor HNF3alpha affects glucose homeostasis and islet glucagon gene expression in vivo. Genes Dev 1999; 13: 495-504.
17. Weinstein DC, Ruiz i Altaba A, Chen WS, Hoodless P, Prezioso VR, Jessell TM et al. The winged-helix transcription factor HNF-3 beta is required for notochord development in the mouse embryo. Cell 1994; 78: 575-588.

18. Shen W, Scearce LM, Brestelli JE, Sund NJ, Kaestner KH. Foxa3 (hepatocyte nuclear factor 3 gamma) is required for the regulation of hepatic GLUT2 expression and the maintenance of glucose homeostasis during a prolonged fast. J Biol Chem 2001; 276 : $42812-42817$.

19. Dai K, Chen R, Ding Y, Niu Z, Fan J, Xu C. Induction of functional hepatocyte-like cells by overexpression of FOXA3 and HNF4alpha in rat bone marrow mesenchymal stem cells. Cells Tissues Organs 2014; 200: 132-140.

20. Seiliez I, Thisse B, Thisse C. FoxA3 and goosecoid promote anterior neural fate through inhibition of Wnt8a activity before the onset of gastrulation. Dev Biol 2006; 290: 152-163.

21. Huang CC, Chuang JH, Chou MH, Wu CL, Chen CM, Wang CC et al. Matrilysin (MMP-7) is a major matrix metalloproteinase upregulated in biliary atresia-associated liver fibrosis. Mod Pathol 2005; 18: 941-950.

22. Narkewicz MR, Kasaragod A, Lucia MS, Pflummer S, Sokol RJ, Stenmark KR. Connective tissue growth factor expression is increased in biliary epithelial cells in biliary atresia. J Pediatr Surg 2005; 40: 1721-1725.

23. Bessho K, Mourya R, Shivakumar P, Walters S, Magee JC, Rao M et al. Gene expression signature for biliary atresia and a role for interleukin-8 in pathogenesis of experimental disease. Hepatology 2014; 60: 211-223.

24. Zhou K, Wang J, Xie G, Zhou Y, Yan W, Pan W et al. Distinct plasma bile acid profiles of biliary atresia and neonatal hepatitis syndrome. J Proteome Res 2015; 14: 4844-4850.

25. Li Z, White P, Tuteja G, Rubins N, Sackett S, Kaestner KH. Foxa1 and Foxa2 regulate bile duct development in mice. J Clin Invest 2009; 119: 1537-1545.

26. Bochkis IM, Rubins NE, White P, Furth EE, Friedman JR, Kaestner KH. Hepatocyte-specific ablation of Foxa2 alters bile acid homeostasis and results in endoplasmic reticulum stress. Nat Med 2008; 14: 828-836.

27. Trapnell C, Pachter L, Salzberg SL. TopHat: discovering splice junctions with RNA-Seq. Bioinformatics 2009; 25: 1105-1111.

28. Trapnell C, Hendrickson DG, Sauvageau M, Goff L, Rinn JL, Pachter L. Differential analysis of gene regulation at transcript resolution with RNA-seq. Nat Biotechnol 2013; 31: 46-53.

29. Trapnell C, Roberts A, Goff L, Pertea G, Kim D, Kelley DR et al. Differential gene and transcript expression analysis of RNA-seq experiments with TopHat and Cufflinks. Nat Protoc 2012; 7: 562-578.

30. Subramanian A, Kuehn H, Gould J, Tamayo P, Mesirov JP. GSEA-P: a desktop application for Gene Set Enrichment Analysis. Bioinformatics 2007; 23: 3251-3253.

31. Huang TH, Zhu MJ, Li XY, Zhao SH. Discovery of porcine microRNAs and profiling from skeletal muscle tissues during development. PLOS ONE 2008; 3: e3225.

32. Tuchweber B, Desmouliere A, Bochaton-Piallat ML, Rubbia-Brandt L, Gabbiani G. Proliferation and phenotypic modulation of portal fibroblasts in the early stages of cholestatic fibrosis in the rat. Lab Invest 1996; 74: 265-278.

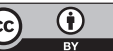

Cell Death and Disease is an open-access journal published by Nature Publishing Group. This work is licensed under a Creative Commons Attribution 4.0 International License. The images or other third party material in this article are included in the article's Creative Commons license, unless indicated otherwise in the credit line; if the material is not included under the Creative Commons license, users will need to obtain permission from the license holder to reproduce the material. To view a copy of this license, visit http://creativecommons.org/licenses/by/4.0/

(C) The Author(s) 2017 